\title{
AUTOMATIC COREGISTRATION FOR MULTIVIEW SAR IMAGES IN URBAN AREAS
}

\author{
Yuming Xiang ${ }^{\mathrm{ab} *}$,Wenchao Kang ${ }^{\mathrm{ab}}$, Feng Wang $^{\mathrm{a}}$, Hongjian You $^{\mathrm{ab}}$ \\ ${ }^{a}$ Key Laboratory of Technology in Geo-spatial Information Processing and Application System, Institute of Electronics, \\ Chinese Academy of Sciences, Beijing, China \\ z199208081010@163.com,xshzhdm@163.com,wfenggucas@126.com,hjyou@mail.ie.ac.cn \\ ${ }^{\mathrm{b}}$ University of Chinese Academy of Sciences, Beijing, China
}

Commission I, WG I/3

KEY WORDS: SAR; Image registration; Urban areas; Building detection

\begin{abstract}
:
Due to the high resolution property and the side-looking mechanism of SAR sensors, complex buildings structures make the registration of SAR images in urban areas becomes very hard. In order to solve the problem, an automatic and robust coregistration approach for multiview high resolution SAR images is proposed in the paper, which consists of three main modules. First, both the reference image and the sensed image are segmented into two parts, urban areas and nonurban areas. Urban areas caused by double or multiple scattering in a SAR image have a tendency to show higher local mean and local variance values compared with general homogeneous regions due to the complex structural information. Based on this criterion, building areas are extracted. After obtaining the target regions, L-shape structures are detected using the SAR phase congruency model and Hough transform. The double bounce scatterings formed by wall and ground are shown as strong L- or T-shapes, which are usually taken as the most reliable indicator for building detection. According to the assumption that buildings are rectangular and flat models, planimetric buildings are delineated using the L-shapes, then the reconstructed target areas are obtained. For the orignal areas and the reconstructed target areas, the SAR-SIFT matching algorithm is implemented. Finally, correct corresponding points are extracted by the fast sample consensus (FSC) and the transformation model is also derived. The experimental results on a pair of multiview TerraSAR images with 1-m resolution show that the proposed approach gives a robust and precise registration performance, compared with the orignal SAR-SIFT method.
\end{abstract}

\section{INTRODUCTION}

With the rapid development of the spaceborne synthetic aperture radar (SAR) sensors, such as TerraSAR-X/TanDEM-X and COSMO-SkyMed, numerous high resolution (HR) SAR images have been widely used in commercial and civil areas (Suri and Reinartz, 2010). As the fundamental task of many image applications, SAR image registration is still a challenging task due to the speckle noise and the side-looking imaging mechanism. In this paper, multiview SAR images refer to two SAR images of the same area obtained from the satellite's ascending and descending orbits. For SAR images in urban or suburban areas, building structures in these areas become visible owing to the high resolution property, offering new possibilities for analyzing and monitoring these areas (Wegner et al., 2010). Consequently, jointly using SAR images from different views of the same area is important and helpful for better building detection, characterization and classification results(Dell' Acqua et al., 2004).

However, visual interpretation of building areas is still challenging due to geometric distortions such as foreshortening, layover, and electromagnetic wave interactions between adjacent targets, together with speckle noise (Zhang et al., 2011). In addition, different building facades may be superimposed since they are on the equidistant line in slant range with respect to the SAR sensor. A lot of efforts have been made to solve these issues in building detection and reconstruction (Xu and Jin, 2007, Ferro et al., 2013, Shahzad and Zhu, 2016), most of them focus on the the line of bright scattering caused by dihedral corner reflector between

\footnotetext{
*Corresponding author
}

ground and building wall, which is considered as the only feature that is nearly invariant to the configuration changes (Thiele et al., 2007). However, few research effort considering the complex building problems was put in the image registration topic. Here, in this paper, we propose an automatic coregistration technique for multiview SAR image in building areas. We only assume the buildings as rectangular and flat models; coregistration of more complex building structures will be explored in future work.

As shown in Figure 1, two multiview SAR images are presented. The bright lines are mainly L-shaped and T-shaped structures. It can be observed that the L-shapes from SAR images of different views provides either the front or the rear facade of the same building. Consequently, the L-shapes correpond to the same building structure appear differently. Since the buidling areas contain plentiful structure information, numerous keypoints can be detected, which may lead to mismatching problems.

In order to solve this problem, an automatic coregistration framework is proposed based on the L-shape detection and reconstruction. Aiming at finding a intermediate description to reduce the difference between the aforementioned L-shapes, we first detect the L-shapes in the reference and sensed images based on a robust ridge detector, then each L-shape is reconstructed to a rectangle structure based on the aforemention assumption and its geometrical property. Finally, the two reconstructed images are matched by a feature-based registration method.

\section{RELATED WORK}

Numerous research efforts have been devoted to SAR image registration. The existing algorithms can be roughly divided into 

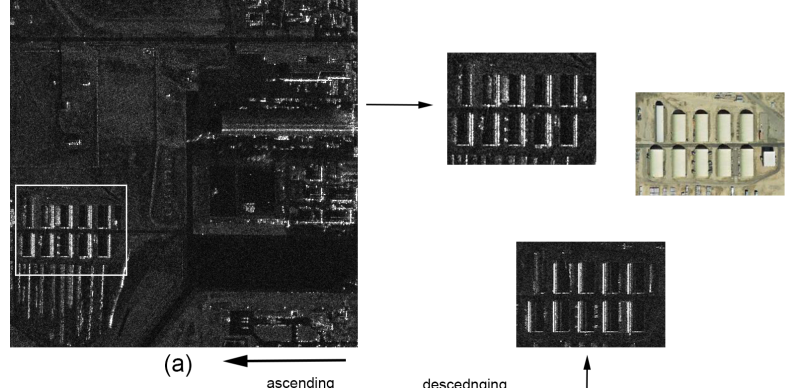

a) ascending

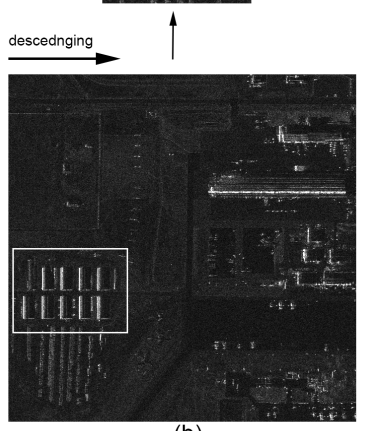

(b)

Figure 1. Two multiview SAR images of the same area and comparsions. (a) Ascending orbit. (b) Descending orbit.

two categories: area-based and feature-based. Area-based techniques first define a template in the sensed image, then search for the optimal correspondence in the reference image using different kinds of similarity measures. The most common used similarity metric is the mutual information as for example used in (Suri and Reinartz, 2010), where SAR (TerraSAR-X) and optical (Ikonos) imagery of urban areas is matched. Though the mutual information has been successfully applied in the coregistration of multispectral or multisensor remote sensing images because of its invariance to non-linear intensity changes (Mellor and Brady, 2005), this similarity measure may lead to local extrema and high computation load (Keller and Averbuch, 2006).

Feature-based techniques first detect distinctive features, such as points, lines and regions, both in the reference and sensed images, then construct descriptors based on the local image neighborhood and attemp to find corresponding features(Hänsch et al., 2016). Among the feature-based methods, the SIFT-like algorithms are the most widely used techniques in SAR image registration due to the efficient performance and invariance to scale, rotation and illumination changes (Dellinger et al., 2015). For example, Schwind et al. (Schwind et al., 2010) proposed SIFT-OCT to extract feature points starting from the second octave. Fan et al. (Fan et al., 2013) improved the matching performance by skipping the dominant orientation assignment when the matching images do not have rotation transformation. Wang et al. (Wang et al., 2012) replaced the Gaussian filter with the Bilateral Filter SIFT (BFSIFT). Dellinger et al. (Dellinger et al., 2015) proposed the SAR-SIFT algorithm specifically dedicated to SAR images by utilizing the ROEWA instead of a differential to calculate a gradient.

However, as no exact keypoint correspondences can be found among the buidling areas, the aforementioned methods are no longer applicable. Only a few researches have considered the coregistration problem in urban areas. They mainly focus on the invariant features in the multiview SAR images, or ignore the variant building structures areas. For example, Acqua et al. (Dell' Acqua et al., 2004) extracted the cross-roads and road junctions from high-resolution urban SAR images, then uses corre- sponding pairs of these features to drive the subsequent coregistration. Though the road junctions are invariant in multiview SAR images, the road detection heavily depends on the performance and parameters selection of detectors, which are still challenging works. Han et al. (Han and Byun, 2015) only found corresponding points in homogeneous regions by extracting heterogeneous regions using a quadtree-based segmentation method. Because keypoints are mainly detected in heterogeneous regions, the corresponding points in homogeneous regions may be not enough to derive a robust transformation model. Wang et al. (Wang and $\mathrm{Zhu}, 2015$ ) used the end points of the building edges where the two point clouds close to match two multiview TomoSAR point clouds in urban area. This method is the most similar to our proposed framework, while it only focuses on multiview TomoSAR point clouds.

\section{METHODOLOGY}

The flowchart of the proposed framework is shown in Figure 2. We do not need to apply a preprocessing method for SAR images, such as speckle reduction, since the techinqiues we used for Lshape detection and image matching are both robust to speckle noise. As a consequence, no information of the original SAR image is lost, and the resolution is not degraded (Wegner et al., 2010).

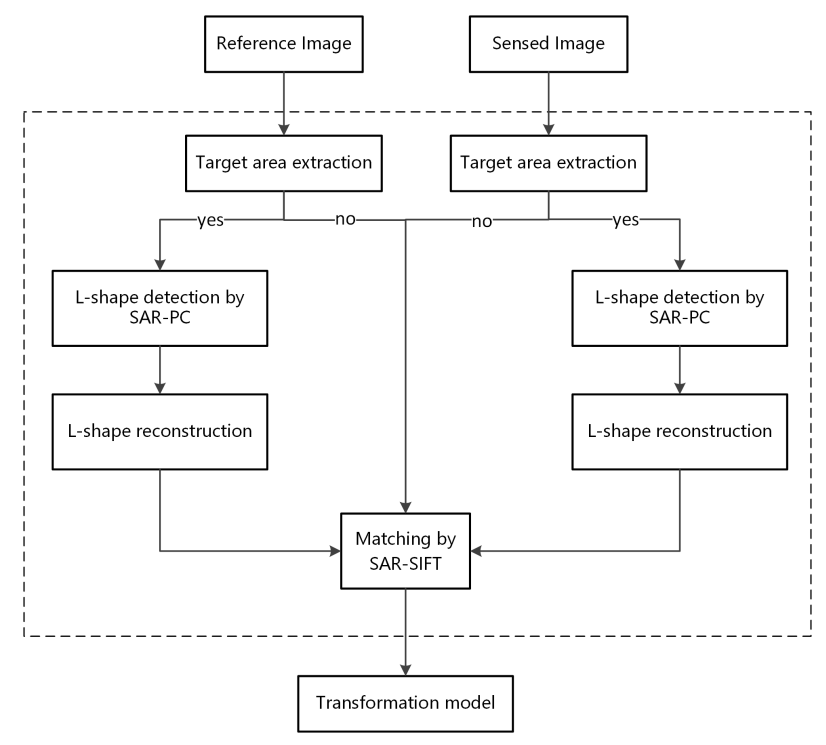

Figure 2. The flowchart of the proposed method.

In the detection process, linear features are first extracted by combining the SAR phase congruency detector (Xiang et al., 2017) and the hysteresis thresholding method. However, the threshols are strongly affected by the template size and image scene, which needs to be manually set, while it is difficult to define a proper threshold to distinguish good linear features from bad ones. In order to solve this issue, an image segmenation method is first applied before detection to extract target area. Building areas caused by double or multiple scattering in SAR images have a tendency to show higher local mean and local variance values compared with general homogeneous regions (Esch et al., 2011). Hence, we use the local mean and local variance of the areas to extrac target regions, which are given as follows:

$$
\mathrm{S}_{\mathrm{i}}=\frac{\mu\left(T_{i}\right)}{\mu(I)}+\frac{\sigma\left(T_{i}\right)}{\sigma(I)},
$$


where $T_{i}$ and $I$ are the pixel sets of the $i$ th region and the entire image, respectively, $\mu$ and $\sigma$ are the mean and variance. We utilize the quadrature-based splitting method in (Han and Byun, 2015). After obtaining target areas, we employ a robust detector based on the phase congrency to extract line features in these areas, followed by the global Hough Transfom. Considering the geometrical property of L-shapes, restrictions on orientation and size are applied to extract the location of L-shape. According to the assumption made in Section 1, L-shapes are recovered to rectangle models. Then the recovered target areas and the homogenous areas are combined to form the recovered image.

We use the SAR-SIFT algorithm (Dellinger et al., 2015) as the matching technique. The SIFT-like algorithms have shown good performance for multi-aspect SAR image registration (Schwind et al., 2010). Among them, the SAR-SIFT is the sate-of-theart method, which contains three major steps: keypoints detection, orientation assignment and descriptor extraction, keypoints matching. First, instead of constructing the Gaussian image pyramid, the algorithm starts with constructing a Harris scale space. Replacing the original gradient with the logarithmic ratio of the exponentially weighted averages (ROEWA) operator (Fjortoft et al., 1998), the gradient by ratio is given as follows:

$$
\begin{aligned}
& G_{h, \alpha}=\log \left(\frac{\sum_{i=-M / 2}^{M / 2} \sum_{j=1}^{N / 2} I(x+i, y+j) e^{-\frac{|i|+|j|}{\alpha}}}{\sum_{i=-M / 2}^{M / 2} \sum_{j=-N / 2}^{-1} I(x+i, y+j) e^{-\frac{|i|+|j|}{\alpha}}}\right) \\
& G_{v, \alpha}=\log \left(\frac{\sum_{i=1}^{M / 2} \sum_{j=-N / 2}^{N / 2} I(x+i, y+j) e^{-\frac{|i|+|j|}{\alpha}}}{\sum_{i=-M / 2}^{-1} \sum_{j=-N / 2}^{N / 2} I(x+i, y+j) e^{-\frac{|i|+|j|}{\alpha}}}\right),
\end{aligned}
$$

where $M$ and $N$ denote the size of the neighborhood, $(x, y)$ stands for the location of the feature point, $\alpha$ is the exponential weight parameter, $I$ represents the pixel intensity. Then the SAR-Harris function is computed at different scales, resulting in the Harris scale space. Local extreme in the scale space are selected as keypoints candidates. Second, dominant orientation is assigned to each keypoint to maintain the rotation invariance, which corresponds to the highest peak in the scale-dependent gradient orientation histogram. A circular neighborhood (size of $6 \sigma)$ and log-polar sectors are employed to construct the descriptor. Third, the keypoint matching stage is similar to the SIFT algorithm, which refers to the Nearest Neighbor Distance Ratio (NNDR) method. Finally, the correct corresponding keypoints are extracted by the fast sample consensus method (Wu et al., 2015), and the transformation model is also derived.

\section{EXPERIMENTS}

In this section, in order to assess the performance of the proposed registration method, a pair of TerraSAR images is used. The images are obtained under ascending and descending orbits, corresponding to two multi-view SAR images. The parameters of the quadrature-based splitting method and the SAR phase congruency method follow their authors' instructions, same as the SAR-SIFT method. Moreover, we use the SAR-SIFT method on the original reference and sensed images as a comparasion.

Figure 3 show the L-shape detection and reconstruction results of the reference and sensed images. It can observed that almost all L-shapes have successfully been detected and reconstructed.

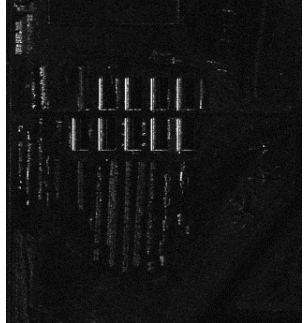

(a)

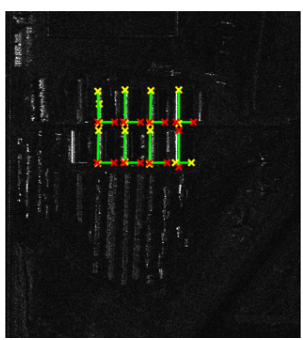

(c)

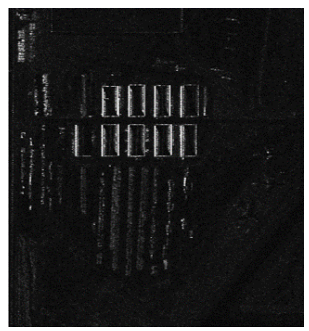

(e)

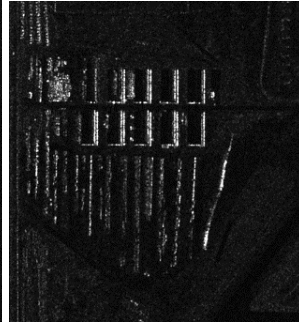

(b)

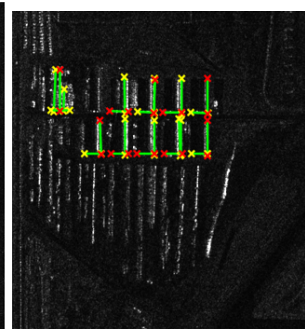

(d)

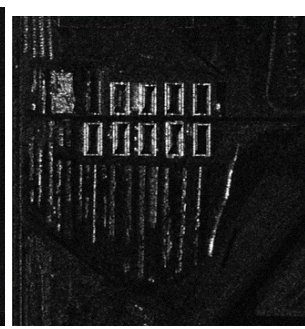

(f)
Figure 3. (a) The reference image (b) L-shape detection of the reference image (c) L-shape reconstruction of the reference image (d) The sensed image (e) L-shape detection of the sensed image (f) L-shape reconstruction of the sensed image

Comparing the two recovered SAR images, the difference of building areas between them has been significantly reduced. Figure 4 illustrate the matching results of the two recovered images and two orignal images. It is clear that the correspondences obtained by our proposed method are correct, while the matched keypoints obtained by the orignal SAR-SIFT correspond to different structure. Since there do not exist repeatable keypoints for the building areas in the orignal reference and sensed images, it is impossible to match these areas. For our proposed method, the building areas have been reconstructed. Consequently, repeatable keypoints can be detected and correctly matched.

\section{CONCLUSION}

In the paper, an automatic and robust coregistration approach for multiview high resolution SAR images is proposed. Aiming at reducing the difference of buidling structures from SAR images of different views, we focus on the L-shapes caused by the doublebounce scattering. First, both the reference image and the sensed image are segmented into two parts, urban areas and nonurban areas. Urban areas caused by double or multiple scattering in a SAR image have higher local mean and local variance values compared with general homogeneous regions due to the complex structural information. Based on this criterion, building areas are extracted and L-shape structures are detected in these areas. For both the orignal areas and the reconstructed areas, the SAR-SIFT 


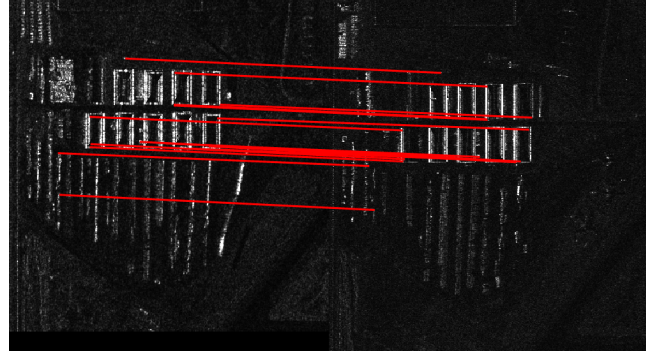

(a)

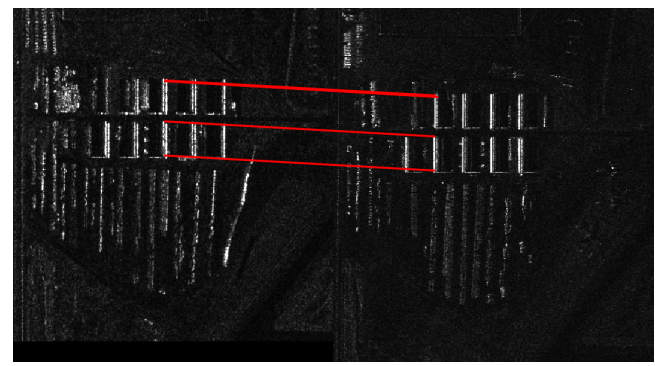

(b)

Figure 4. The matching results. (a) Our proposed method (b) The original SAR-SIFT

matching algorithm is employed. Finally, correct corresponding points are extracted by the fast sample consensus (FSC) and the transformation model is also derived. Experimental results on a pair of multiview TerraSAR images show that the proposed approach gives a robust and precise registration performance, compared with the orignal SAR-SIFT method.

\section{ACKNOWLEDGEMENTS}

This work was supported by the Fundamental Research Project under Grant 41-Y20A13-9001-15/16.

\section{REFERENCES}

Dell' Acqua, F., Gamba, P. and Lisini, G., 2004. Coregistration of multiangle fine spatial resolution sar images. IEEE Geoscience and Remote Sensing Letters 1(4), pp. 237-241.

Dellinger, F., Delon, J., Gousseau, Y., Michel, J. and Tupin, F., 2015. Sar-sift: a sift-like algorithm for sar images. IEEE Transactions on Geoscience and Remote Sensing 53(1), pp. 453-466.

Esch, T., Schenk, A., Ullmann, T., Thiel, M., Roth, A. and Dech, S., 2011. Characterization of land cover types in terrasar-x images by combined analysis of speckle statistics and intensity information. IEEE Transactions on Geoscience and Remote Sensing 49(6), pp. 1911-1925.

Fan, B., Huo, C., Pan, C. and Kong, Q., 2013. Registration of optical and sar satellite images by exploring the spatial relationship of the improved sift. IEEE Geoscience and Remote Sensing Letters 10(4), pp. 657-661.

Ferro, A., Brunner, D. and Bruzzone, L., 2013. Automatic detection and reconstruction of building radar footprints from single vhr sar images. IEEE Transactions on Geoscience and Remote Sensing 51(2), pp. 935-952.

Fjortoft, R., Lopes, A., Marthon, P. and Cubero-Castan, E., 1998. An optimal multiedge detector for sar image segmentation. IEEE Transactions on Geoscience and Remote Sensing 36(3), pp. 793 802.
Han, Y. and Byun, Y., 2015. Automatic and accurate registration of vhr optical and sar images using a quadtree structure. International Journal of Remote Sensing 36(9), pp. 2277-2295.

Hänsch, R., Hellwich, O. and Tu, X., 2016. Machine-learning based detection of corresponding interest points in optical and sar images. In: Geoscience and Remote Sensing Symposium (IGARSS), 2016 IEEE International, IEEE, pp. 1492-1495.

Keller, Y. and Averbuch, A., 2006. Multisensor image registration via implicit similarity. IEEE transactions on pattern analysis and machine intelligence 28(5), pp. 794-801.

Mellor, M. and Brady, M., 2005. Phase mutual information as a similarity measure for registration. Medical image analysis 9(4), pp. 330-343.

Schwind, P., Suri, S., Reinartz, P. and Siebert, A., 2010. Applicability of the sift operator to geometric sar image registration. International Journal of Remote Sensing 31(8), pp. 1959-1980.

Shahzad, M. and Zhu, X. X., 2016. Automatic detection and reconstruction of 2-d/3-d building shapes from spaceborne tomosar point clouds. IEEE Transactions on Geoscience and Remote Sensing 54(3), pp. 1292-1310.

Suri, S. and Reinartz, P., 2010. Mutual-information-based registration of terrasar-x and ikonos imagery in urban areas. IEEE Transactions on Geoscience and Remote Sensing 48(2), pp. 939949.

Thiele, A., Cadario, E., Schulz, K., Thonnessen, U. and Soergel, U., 2007. Building recognition from multi-aspect high-resolution insar data in urban areas. IEEE Transactions on Geoscience and Remote Sensing 45(11), pp. 3583-3593.

Wang, S., You, H. and Fu, K., 2012. Bfsift: A novel method to find feature matches for sar image registration. IEEE Geoscience and remote sensing letters 9(4), pp. 649-653.

Wang, Y. and Zhu, X. X., 2015. Automatic feature-based geometric fusion of multiview tomosar point clouds in urban area. IEEE Journal of Selected Topics in Applied Earth Observations and Remote Sensing 8(3), pp. 953-965.

Wegner, J. D., Auer, S. and Soergel, U., 2010. Extraction and geometrical accuracy of double-bounce lines in high resolution sar images. Photogrammetric Engineering \& Remote Sensing 76(9), pp. 1071-1080.

Wu, Y., Ma, W., Gong, M., Su, L. and Jiao, L., 2015. A novel point-matching algorithm based on fast sample consensus for image registration. IEEE Geoscience and Remote Sensing Letters 12(1), pp. 43-47.

Xiang, Y., Wang, F., Wan, L. and You, H., 2017. Sar-pc: Edge detection in sar images via an advanced phase congruency model. Remote Sensing 9(3), pp. 209.

Xu, F. and Jin, Y.-Q., 2007. Automatic reconstruction of building objects from multiaspect meter-resolution sar images. IEEE Transactions on Geoscience and Remote Sensing 45(7), pp. 2336-2353.

Zhang, F., Shao, Y., Zhang, X. and Balz, T., 2011. Building 1shape footprint extraction from high resolution sar image. In: $U r$ ban Remote Sensing Event (JURSE), 2011 Joint, IEEE, pp. 273276. 